\title{
Selections that isolate recombinant mitochondrial genomes in animals
}

\author{
Hansong Ma, Patrick H O'Farrell* \\ Department of Biochemistry and Biophysics, University of California, San Francisco, \\ San Francisco, United States
}

\begin{abstract}
Homologous recombination is widespread and catalyzes evolution. Nonetheless, its existence in animal mitochondrial DNA is questioned. We designed selections for recombination between co-resident mitochondrial genomes in various heteroplasmic Drosophila lines. In four experimental settings, recombinant genomes became the sole or dominant genome in the progeny. Thus, selection uncovers occurrence of homologous recombination in Drosophila mtDNA and documents its functional benefit. Double-strand breaks enhanced recombination in the germline and revealed somatic recombination. When the recombination partner was a diverged Drosophila melanogaster genome or a genome from a different species such as Drosophila yakuba, sequencing revealed long continuous stretches of exchange. In addition, the distribution of sequence polymorphisms in recombinants allowed us to map a selected trait to a particular region in the Drosophila mitochondrial genome. Thus, recombination can be harnessed to dissect function and evolution of mitochondrial genome.

DOI: 10.7554/eLife.07247.001
\end{abstract}

*For correspondence: ofarrell@ cgl.ucsf.edu

Competing interests: The authors declare that no competing interests exist.

Funding: See page 14

Received: 28 February 2015

Accepted: 01 August 2015

Published: 03 August 2015

Reviewing editor: Jodi Nunnari, University of California, Davis, United States

Copyright $\mathrm{Ma}$ and O'Farrell. This article is distributed under the terms of the Creative

Commons Attribution License, which permits unrestricted use and redistribution provided that the original author and source are credited.

\section{Introduction}

Homologous recombination operates in organisms from bacteriophage to human. This includes the mitochondrial genomes in many plant and fungal species (Rank and Bech-Hansen, 1972; Dujon et al., 1974; André et al., 1992; Zassenhaus and Denniger, 1994; Shedge et al., 2007). Nonetheless, there is very little support for recombination in animal mitochondria (Elson et al., 2001; Berlin et al., 2004; Hagström et al., 2013); lack of an identified mitochondrial RecA homolog, evidence of continuous lineages of mitochondrial haplotypes and a failure to detect recombinants in propagated heteroplasmic lines are taken as indications that it does not occur.

Despite evidence arguing against recombination of animal mitochondrial genomes, a variety of exceptional reports suggest that it can occur. The remarkable 'double uniparental inheritance' pattern of mitochondrial genomes in some bivalve mollusks has been associated with rare recombination events on at least an evolutionary time scale (Ladoukakis and Zouros, 2001; Ladoukakis et al., 2011). One human patient was reported to carry recombinant genomes (Kraytsberg, 2004), and there have been reports of recombinant mitochondrial genotypes in some species like lizard and fish (Guo et al., 2006; Ciborowski et al., 2007; Ujvari et al., 2007), but these reports are based on single individuals without documentation of parents or origin of the genomes presumed to have recombined. Given opposing observations, such as the introgression of intact genomes from one species into another (Solignac, 2004), it is not clear whether the cases reported are exceptional, or whether we have simply lacked the experimental power to directly demonstrate recombination in animals.

Recombination between small regions of nonallelic homology has been proposed to underlie deletion and insertion mutations (Mita et al., 1990; Bacman et al., 2009; Fukui and Moraes, 2009). However, when precise, recombination can generate favorable combinations of alleles, which, when coupled to the action of purifying selection, could increase in abundance to restore function 
eLife digest Animals store the main part of their DNA-including all of the genes that are required to build and maintain an individual-inside their cells in a structure called the nucleus. Most of the information stored in the DNA is stored in duplicate, with one copy inherited from the individual's mother via the egg, and the other from the father via the sperm. This duplicate storage allows a very important damage repair process to occur, where undamaged sequences in one copy can be used to repair damage in the other. This process of homologous repair uses mechanisms that are also used in another important genetic process. When sperm and egg cells are formed, the parental DNA goes through a process called homologous recombination, in which DNA molecules are cut and reassembled into new arrangements. This recombination process 'shuffles' genetic combinations, making every individual unique-a process of great evolutionary importance that allows natural selection to act on distinct traits.

The structures inside cells that generate energy-called mitochondria-also contain DNA, which is inherited only from mothers. Little is known about whether recombination is possible in the mitochondrial DNA of animals.

$M a$ and O'Farrell used genetics techniques to investigate recombination in the mitochondria of fruit flies. One experiment tracked how a mutation that makes flies less healthy at high temperatures spread as flies were bred for several generations. When the mutation was associated with a mitochondrial genome that had a strong drive towards replication, the mutation became more widespread over time, and in most cases, this eventually resulted in the mutation killing the flies. In rare cases, however, a few flies survived, giving rise to a healthy population. Molecular analyses revealed that, in these survivors, the defective genome had recombined with the other mitochondrial DNA to produce a new genome that lacked the mutation but retained the high replicative drive. This new recombinant genome worked normally and was able to resist the spread of the defective genomes.

In addition, by artificially cutting mitochondrial DNA, Ma and O'Farrell show that such 'doublestrand breaks' lead to recombination, signaling a role for homologous repair in the repair of damaged, in this case broken, DNA. Recombination is also possible between the mitochondrial DNA of two different fruit fly species, and this recombination process can assemble long stretches of DNA.

Now that the recombination of animal mitochondrial DNA is known to be possible, future work will be required to understand how it works and how it affects evolution. DOI: 10.7554/eLife.07247.002

(Muller, 1932). These positive or negative impacts on gene function could influence evolution, the behavior of disease mutations, and the age-associated degenerative changes of the mitochondrial genome.

Until recently, several factors have hindered detection of recombination of mitochondrial sequences. Chief among these, uniparental inheritance largely limits exposure of mitochondrial genomes to sibling genomes differing only at newly mutated sites (Birky, 1995; DeLuca and O'Farrell, 2012; Sato and Sato, 2013). Additionally, rare recombinant genomes can be difficult to detect: they can be stochastically lost during the random segregation, and if transmitted, they can be hard to track amid the chaotically segregating genomes. Finally, there are few markers suitable for design of conditions that would select for a rare recombinant genome.

Previous work in Drosophila showed that germline expression of a restriction enzyme targeted to mitochondria results in potent selection against mitochondrial genomes carrying a cognate cleavage site (Xu et al., 2008). Using this selection, a number of variant genomes lacking a particular site have been selected. In addition to removing a restriction site, these selected changes often also alter an encoded gene product (Figure 1A). One of the variants that lost a Xhol site is a temperature-sensitive lethal mutation of $\mathrm{mt}: \mathrm{Col}$ that can be counter-selected at high temperature (Hill et al., 2014; Ma et al., 2014). Moreover, one can transfer cytoplasm between early Drosophila embryos of different mitochondrial genotypes to create heteroplasmic lines that carry both the recipient and donor genomes for multiple generations (Matsuura et al., 1989; Ma et al., 2014). In addition to these tools, characterization of diverged mitochondrial genomes has revealed marked differences in their 
A

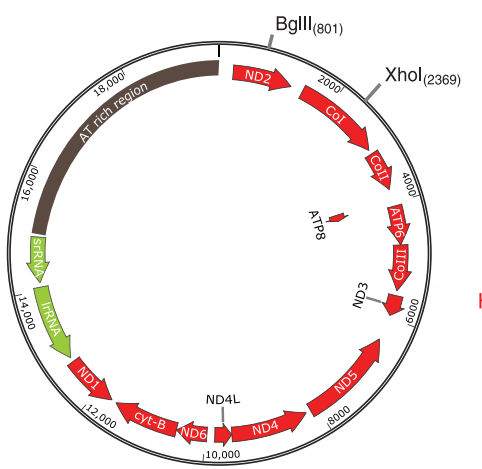

B

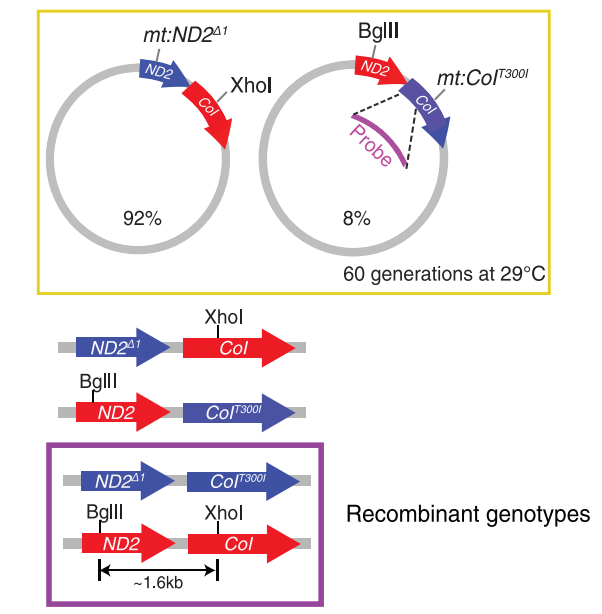

BglII

$w t$

W $\underline{M} \underline{L} \underline{S} \underline{S}$ $\frac{W}{\text { tgaatattaagatct }}$

$m t: N D 2^{\Delta 1} \quad \frac{\mathrm{W}}{\operatorname{tga}} \quad \frac{\mathrm{S}}{--------\operatorname{tct}}$

Healthy with minor defects.
Xhol

wt $\quad \frac{\mathrm{T}}{\text { actcgagct }} \frac{\mathrm{R}}{\mathrm{A}}$

$m t: C o / R 3010 \quad \frac{\mathrm{T}}{\text { actcaagct }}$

Male sterile, but otherwise healthy.

$m t: \mathrm{Col}^{\text {T300I }} \quad \frac{\mathrm{I}}{\text { attcga }} \frac{\mathrm{R}}{\mathrm{gct}}$

At $29^{\circ} \mathrm{C}$ : lethal; adults die after 4 days eggs develop to a late pupal stage.

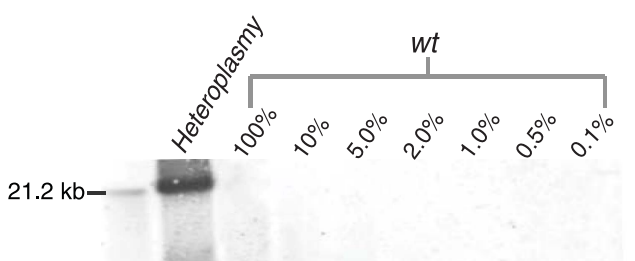

$5.1 \mathrm{~kb}-$

$\mathrm{kb}-$

$2.0 \mathrm{~kb}-$

$1.5 \mathrm{~kb}-$

Figure 1. Without selection, no recombination was detected in a stable heteroplasmic line after 60 generations. (A) Mutants at the Bglll and Xhol sites of mtDNA used in this study (Xu et al., 2008; Ma et al., 2014). The mitochondrial genome of Drosophila resembles that of mammals. It has little intergenic spacing, and encodes 13 polypeptides, all of which are involved in oxidative phosphorylation as well as 22 tRNAs and two rRNAs required for mtDNA translation. A single non-coding region ( $\sim 5 \mathrm{~kb}$ ) called the 'AT-rich region' (dark brown) as it contains $>90 \% \mathrm{~A}$ and $\mathrm{T}$ residues includes origins of replication and some repeated sequences of unknown function (Lewis et al., 1994). The genome contains one Bglll and one Xhol site in the coding regions of $m t$ : ND2 and mt:Col, respectively. (B) No wild-type genome was detected by Southern blotting analysis in heteroplasmic lines where $\mathrm{mt}: \mathrm{ND}^{\text {del1 }}$ and $\mathrm{mt}: \mathrm{Col}^{\text {T300I }}$ were maintained in the same population for more than 60 generations at $29^{\circ} \mathrm{C}$. In the heteroplasmic lane, 40 adults were sacrificed and their mtDNA were cut with both Bglll and Xhol, and probed by a DIG-labeled sequence that hybridizes to mt1579-mt2369. The sensitivity of the Southern analysis was measured by loading a series of dilutions of wild-type mtDNA cut with both enzymes from 40 adult flies.

DOI: 10.7554/eLife.07247.003

abilities to compete for transmission when combined in heteroplasmic combinations, a feature that we have been able to use as another selectable trait. In this work, we have combined these tools to create a powerful system in which we can test for the existence of homologous recombination and select for recombinant genomes.

Here, we provide clear evidence for homologous genetic exchange between Drosophila mitochondrial genomes under various conditions. The complete genomic sequence of parental and recombinant molecules details exchange events, and several recombinants are shown to involve transfer of a substantial segment of sequence from one genome to the other. We also show that exchange is stimulated by double-strand breaks (DSBs), as is recombination in many systems. Importantly, the success of the selections that we have applied shows that production of favorable combinations of alleles by recombination, even if rare, can have a profound benefit. 


\section{Results}

\section{Direct screening for recombinant genomes in a heteroplasmic line}

We used Southern analysis to test for recombination between genomes distinguished by differences in restriction sites. A line heteroplasmic for $\mathrm{mt}: \mathrm{ND}^{\text {del1, }}{ }^{\text {, which lacks the Bglll site, and mt:Col }}{ }^{\mathrm{T300l}}$, which lacks the Xhol site, carries both genomes stably at $29^{\circ} \mathrm{C}$ (Figure $\left.1 \mathrm{~B}\right)$ (Ma et al., 2014). Each of the genomes of this heteroplasmic line has a defect complemented by the other so that the line is healthy but neither genomes is lost, a balancing selection (Ma et al., 2014). Recombination should produce wild-type genomes distinguished by the presence of both restriction sites and consequent production of a 1 1.6-kb fragment upon cutting with both Bglll and Xhol. Even after maintenance of this line for more than 60 generations, this $1.6 \mathrm{~kb}$ band was not detected (Figure 1B). Reconstruction shows that this Southern assay can detect recombinant molecules at the level of 1 in 1000 (Figure 1B). Thus, like related experiments by others (Hagström et al., 2013), this physical assay failed to detect recombination. We conclude that recombination in this situation is not frequent (Sato et al., 2005).

\section{Selection reveals recombination between mitochondrial genomes}

We then developed methods to genetically select for recombinant mitochondrial genomes in the hopes that such approaches would both detect its occurrence and allow isolation of the recombination product. To give recombinant genomes an advantage, we produced a heteroplasmic line wherein one genome was compromised by a temperature-sensitive mutation and the other was compromised in its ability to compete for transmission. A temperature-sensitive genome with two mutations, mt:ND2 ${ }^{\text {del1 }}+m t: \mathrm{Col}^{\text {T30ol, }}$, was introduced into a strain with a genome named ATP6[1] (Celotto et al., 2006, 2011). High temperature, $29^{\circ} \mathrm{C}$, selected against the $\mathrm{mt}: \mathrm{Col}^{\top 300 l}$ allele, and in previously analyzed heteroplasmic lines where the partner genome was a closely related wild-type genome, this selection resulted in a multi-generational decline of the temperature-sensitive genome and its eventual elimination (Ma et al., 2014). To our surprise, when in competition with the ATP6[1] genome, which is distinguished by numerous sequence polymorphisms and a shorter AT-rich region, the temperature-sensitive genome displaced the ATP6[1] genome over a few generations at $29^{\circ} \mathrm{C}$, even though the flies homoplasmic for ATP6[1] are relatively healthy and apparently more robust than $m t: N D 2^{\text {del1 }}+m t: \mathrm{Col}^{\text {T300l }}$ flies at both temperatures. The decline of ATP6[1] leaves the temperaturesensitive genome without complementing $m t$ :Col activity, and the entire population dies after several generations (Figure 2A).

To select for possible recombinant genomes, we followed five independently established lines with a high starting proportion of ATP6[1] genomes (50-90\%) at $29^{\circ} \mathrm{C}$. Initially, all the lines were healthy and grew productively, but with decline in ATP6[1] abundance, the health of the lines held at $29^{\circ} \mathrm{C}$ declined abruptly after five or six generations and died out within the next few generations. One line went through a similar crisis with diminished survival, recovered and continued to produce viable progeny in subsequent generations. Southern analysis showed emergence of a new genotype, which was cut by Xhol (like the ATP6[1] genome), but had a long AT-rich region (like the double mutant) (Figure 2B). To map recombination sites, we sequenced the parental and recombinant genomes using the PacBio single molecule real-time sequencing technique (SMRT), whose long reads gave us unambiguous sequence that included the repeats of the $5 \mathrm{~kb}$ highly AT-rich noncoding region (Figure 1A and Figure 2). Complete sequences of these genomes revealed that the two parental genomes differed by more than 100 SNPs plus $>20$ indels, and the ATP6[1] genome lacked $\sim 1.6 \mathrm{~kb}$ of the AT-rich region that was present in the $m t: N D 2^{d e l 1}+m t: C o{ }^{T 300}$ genome (Figure $2 \mathrm{C}$ and Figure 2-figure supplement 1A). The recombinant genome was the result of an exchange of a large continuous segment to produce an $\sim 60 \% / 40 \%$ chimera of the ATP6[1] and mt:ND2 ${ }^{\text {del } 1}+\mathrm{mt}^{\mathrm{CO}} \mathrm{Col}^{\mathrm{T300}}$ genomes that lacks the $\mathrm{mt}: \mathrm{ND} 2$ and $\mathrm{mt}$ :Col mutations but contains the non-coding region of the $\mathrm{mt}$ : $\mathrm{ND} 2^{\text {del1 }}+\mathrm{mt}: \mathrm{Col}^{\mathrm{T} 300 \mathrm{l}}$ genome (Figure $2 \mathrm{C}$ ).

Southern analysis also showed the abundance of the recombinant genome at later stages of the selection. After generation 6, the original ATP6[1] genome was no longer detected (Figure 2B), and a new heteroplasmic line was formed with the other parental genome mt:ND2 ${ }^{\text {del } 1}+m t: \mathrm{Col}^{\mathrm{T3} 300 \mathrm{l}}$ and the recombinant genome. This line was viable at $29^{\circ} \mathrm{C}$ as the temperature-sensitive defect of the double mutant is complemented by the ATP6[1] mt:Col of the recombinant genome (Figure 2D). Over subsequent generations, a multigenerational selection for function caused an increase in the 
A

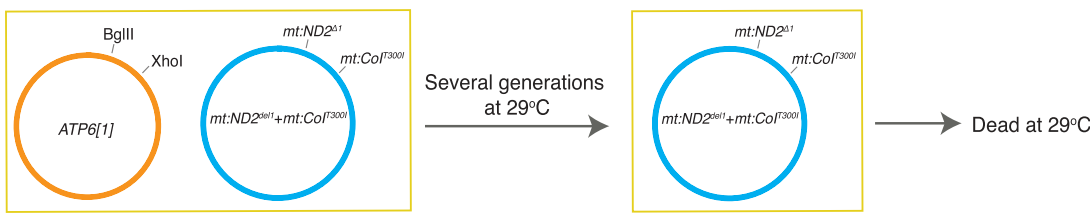

B
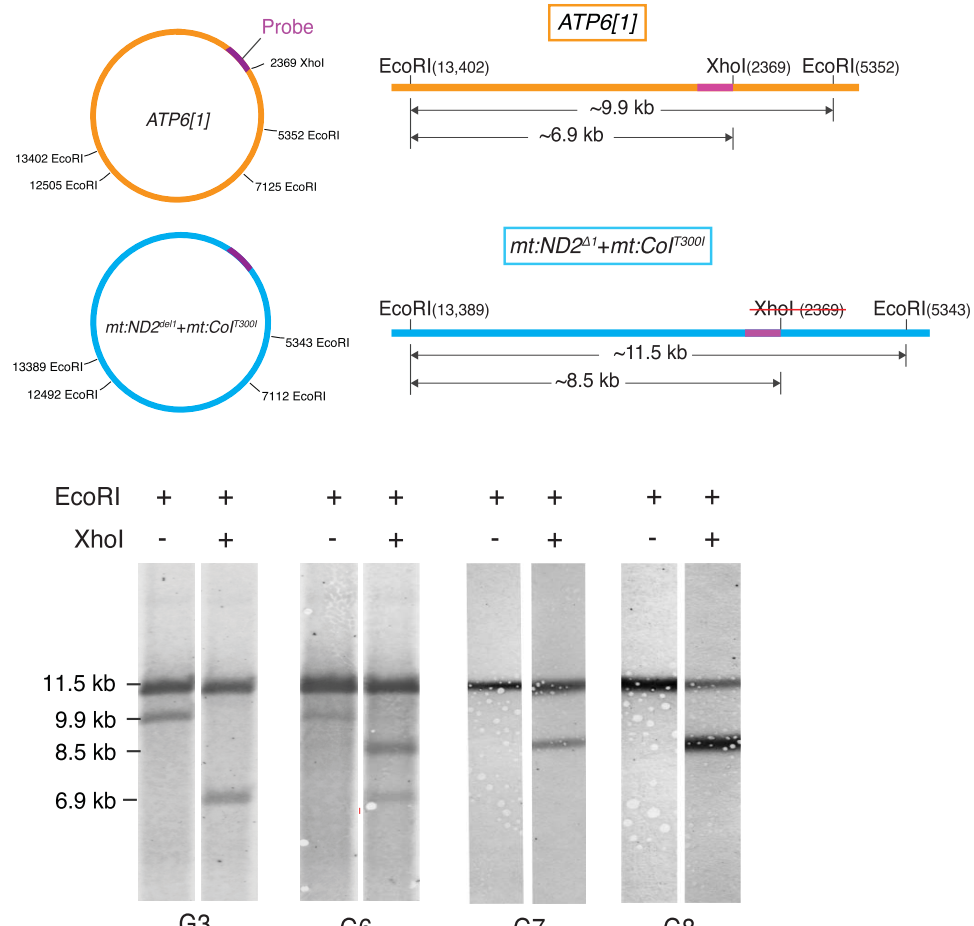

C

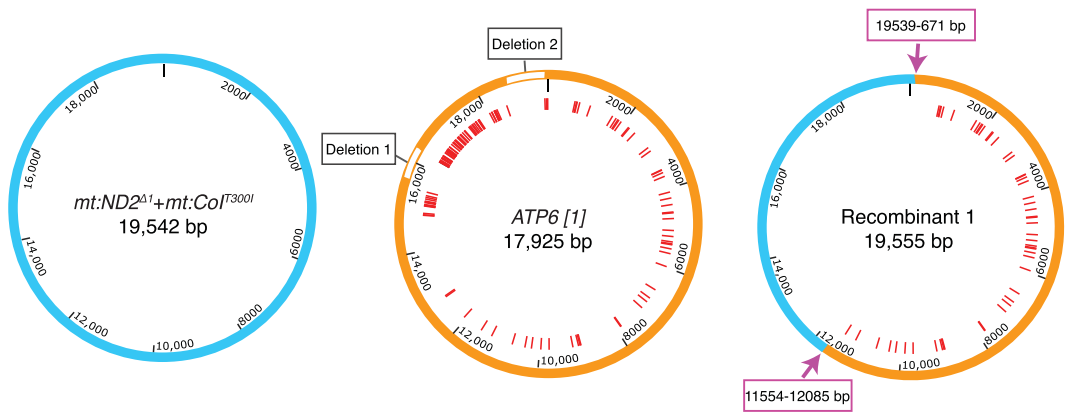

D

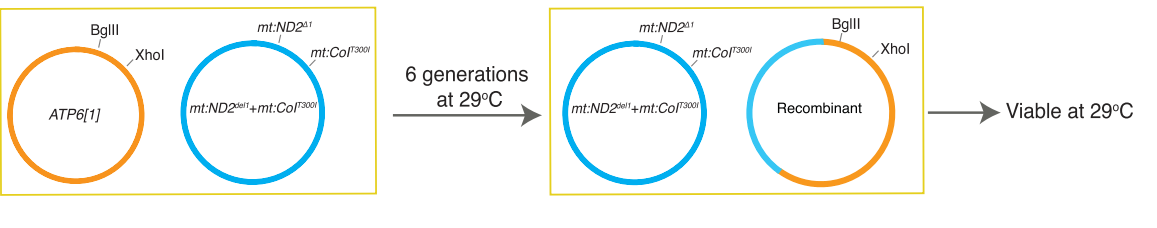

Figure 2. Selection revealed homologous recombination in a heteroplasmic line containing the ATP6[1] genome and the temperature-sensitive double-mutant: $m t: N D 2^{\text {del1 }}+m t: C o l T^{3001}$. (A) The abundance the ATP6[1] genome declined when co-resident with $m t: N D 2^{\text {del1 }}+m t: \mathrm{Col}^{T 3001}$ at $29^{\circ} \mathrm{C}$. After several generations, the flies at $29^{\circ} \mathrm{C}$ started to Figure 2. continued on next page 
Figure 2. Continued

die. (B) A combination of a restriction fragment length polymorphism and a restriction site difference reveals the emergence of a recombinant genome. mtDNA isolated from 40 adults from each generation was cut with EcoRI in the presence or absence of Xhol. The schematics show the distribution of the EcoRI and Xhol sites on the whole parental genomes (left) and a detail of the largest EcoRI fragment with the position (purple bar) of a hybridization probe (center). Southern analysis shows single and double cut samples taken at different generations during the selection. Only the two parental bands were detected early, from G0 to G5 (shown for G3). From G6 onward, a third EcoRl fragment appeared that had a length characteristic of the $m t: N D 2^{\text {del1 }}+m t: C o{ }^{T 300 l}$ genome but with a Xhol site. By G7, the ATP6[1] specific fragment was not detected while a new apparently recombinant genome dominated the population. (C) Detailed maps of three genomes sequenced by PacBio SMRT. Red lines indicate mismatches between ATP6[1] and mt:ND2 ${ }^{\text {del1 }}+\mathrm{mt}: \mathrm{Col}^{\text {T300l }}$ sequences. The ATP6[1] genome also lacks $\sim 1.6 \mathrm{~kb}$ of the AT-rich region (two type I repeats and two type II repeats, see Figure 2-figure supplement 1A for details). Pink arrows indicate approximate points of exchange with the given range defined by the nearest neighboring polymorphisms (see deposited full sequences in GenBank as KT174472, KT174473 and KT174474). (D) Proposed progression leading to the recombinant. The original ATP6[1] genome is still displaced, but the newly emerged recombinant competes effectively and persists. By generation 6, the abundance of the recombinant genome is sufficient to complement the temperature-sensitive genome so that some viable flies sustain the line. Over subsequent generations at $29^{\circ} \mathrm{C}$, the recombinant genome increases in relative abundance because of selection against the temperature-sensitive genome.

DOI: 10.7554/eLife.07247.004

The following figure supplement is available for figure 2 :

Figure supplement 1. mtDNA maps for the two parental genotypes (ATP6[1], mt:ND2del1 $+m t: C o l T^{3001}$ ) and two other recombinants.

DOI: 10.7554/eLife.07247.005

proportion of the recombinant genome (Figure 2B), showing that it had lost the transmission disadvantage of the parental ATP6[1] genome. This led us to conclude that the ability to compete for transmission is localized to the sequences of the recombinant that came from the temperaturesensitive genome. This includes the entire AT-rich regulatory region and some flanking sequences distinguished by three SNPs (Figure 2C).

We later isolated two other recombinant genomes by following another 46 heteroplasmic lines as above. Both recombinants had a size $(\sim 19.5 \mathrm{~kb})$ similar to the temperature-sensitive genome, implying that $m t: N D 2^{d e l 1}+m t: C o{ }^{T 300 l}$ was the source of the regulatory region. By sequencing the coding region, we show that one recombinant has the entire coding sequence of the ATP6[1] genome, whereas the other contains a much smaller segment of ATP6[1] extending at least from mt671 to $\mathrm{mt5978}$, with the rest of the coding region belonging to the $\mathrm{mt}: \mathrm{ND}^{\text {del1 }}+\mathrm{mt}: \mathrm{Col}^{\mathrm{T} 300 \mathrm{l}}$ genome (Figure 2-figure supplement 1B). We conclude that our selection has isolated recombinant genomes including extensive stretches of sequence originating from the parental genomes and exhibiting functional traits of these parental genomes.

\section{Homologous exchange upon introduction of reciprocal DSBs}

Normal meiotic recombination is induced by DSBs and studies in many contexts have suggested that DSBs, whether experimentally produced or secondary to DNA damage, greatly stimulates recombination. Indeed, DSBs are thought to be the key initiators of homologous exchange. To test the importance of DSBs for mtDNA recombination, we set up a condition to select for recombination in conjunction with restriction cutting of the mtDNA.

We had produced genomes that lacked the Xhol site, mt:Col ${ }^{R 301 Q}$, or the Bglll site, mt:ND2 ${ }^{\text {del1 }}$. While each genome is resistant to one enzyme, expression of mito-Bglll and mito-Xhol simultaneously in the germline should cut either of these genomes. Indeed, germline expression of both enzymes in flies that were homoplasmic for either $m t: N D 2^{d e l 1}$ or $m t: C o l{ }^{R 3010}$ led to sterility in females (Figure 3A). As previously shown, resistance ought to emerge at a low frequency due to mutations at the second restriction site (Xu et al., 2008). Indeed, upon selection $\sim 1 \%$ of the females were weakly fertile, giving a few escaper $\mathrm{F} 1$ progeny harboring a variety of mutant alleles that inactivate the remaining restriction site. In contrast, even though the combination of enzymes was able to select against both genotypes, when flies were heteroplasmic for the two genomes (in a 50:50 ratio), fertile females were very 
A

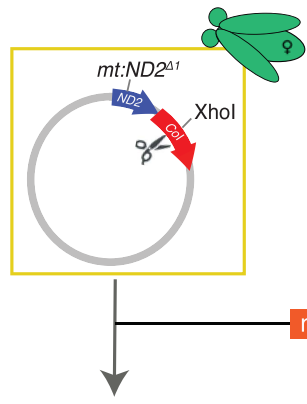

$<1 \%$ female fertile

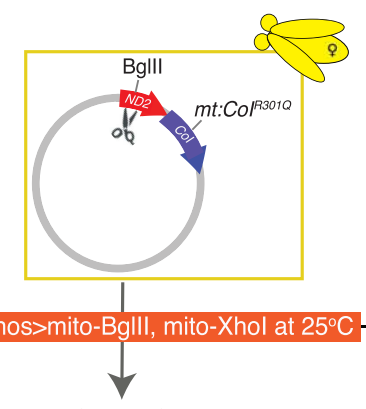

$<1 \%$ female fertile

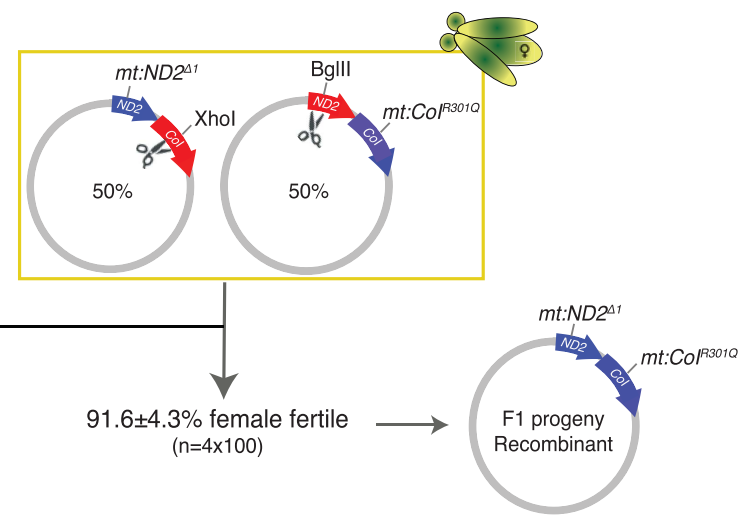

B

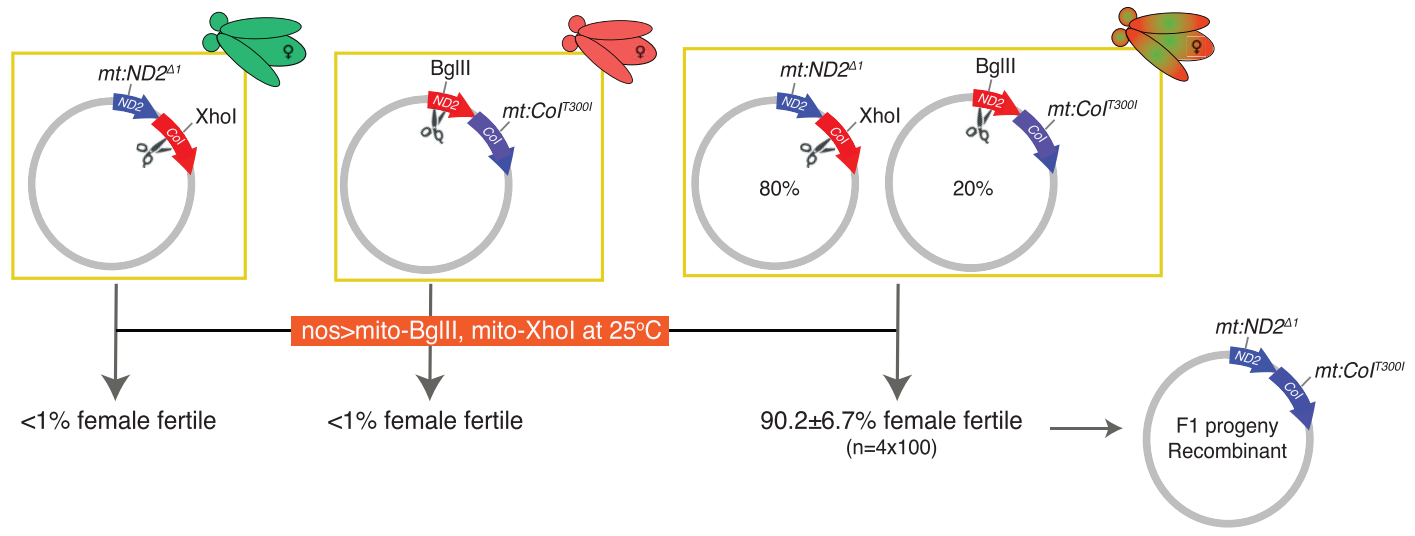

Figure 3. Introducing DSBs into both parental genomes vastly induces homologous recombination in two heteroplasmic lines. (A) Expression of both mito-Bglll and mito-Xhol enzymes in the germline effectively sterilizes most females carrying either mt:ND2 ${ }^{\text {del1 }}$ or mt:Col ${ }^{R 3010}$ genome, resistant to only one of the enzymes, but resistant progeny appear at a much higher frequency if the female carries both types of sensitive genomes. The resistant progeny from the heteroplasmic parent are homoplasmic for a newly generated recombinant genotype carrying both parental alleles: mt:ND2 ${ }^{\text {del1 }}+$ mt:Col ${ }^{\text {R3010 }}$. (B) The same level of rescue was observed when a different heteroplasmic line was used, which had a different starting ratio of two parental genomes. Results are means \pm SD ( $n=4 \times 100$ for each heteroplasmic line).

DOI: 10.7554/eLife.07247.006

frequent (>90\%), and most (60\%) were as fecund as wild type. PCR, DNA sequencing, and phenotypic analysis showed that these progeny carried a single genome with the restriction-resistant alleles found in the two parents (Figure 3A).

A second heteroplasmic line where the $\mathrm{mt}: \mathrm{Col}^{\mathrm{R3010}}$ allele was replaced with the $\mathrm{mt}: \mathrm{Col}^{\mathrm{T3} 300}$ allele gave a similar frequency of recombinant progeny, even though the starting ratio for the two parental genomes was not equal (Figure 3B). The resulting recombinant lines exhibited the phenotypes expected for the input alleles. For instance, $m t: N D 2^{d e l 1}+m t: C o l^{R 3010}$ flies were male sterile, and $m t:$ $N D 2^{\text {del1 }}+m t: \mathrm{Col}^{\text {T30ol }}$ flies were temperature lethal.

The success of a second and very different selection confirms that homologous exchange between mitochondrial genomes can occur. Most females exposed to the restriction enzyme selection were fertile, while in the selection without DSBs (above, Figure 2), the recombinant emerged from a population over several generations of selection. The difference suggests that the frequency of recombination upon expression of the two restriction enzymes is much higher. We propose that DSBs produced by restriction enzyme cutting induces exchange as well as selecting for the products that are resistant to cutting.

To test whether recombination could also occur in somatic tissues, we examined the consequence of expressing both restriction enzymes in the developing eye under the control of ey:GAL4 driver. The high level of expression that occurs at $29^{\circ} \mathrm{C}$ results in pupal lethality (headless phenotype) in $99 \%$ flies 
that are homoplasmic for mitochondrial genomes mutant at only one site and the few survivors are eyeless or have a small eye phenotype. Similar expression in flies heteroplasmic for the two mutant genomes gave survivors at $10 \%$ with most survivors showing well-developed eyes (Supplementary file 1), suggesting that recombination of mtDNA also occurred in somatic tissues exposed to the double restriction enzyme selection. Somatically, active recombination could impact the stability of the mitochondrial genome in the soma, a factor thought to be important in aging (Cortopassi et al., 1992; Liu et al., 1998; Cao et al., 2001; Bender et al., 2006).

\section{Recombination upon introduction of a single DSB}

Given the high frequency of recovered recombinants when both genomes of a heteroplasmic strain were cut, we asked whether we could detect recombination if DSBs were introduced into only one genome. To do this, we made a heteroplasmic line containing the wild-type genome and the temperature-sensitive double-mutant $\mathrm{mt}: \mathrm{ND}^{\mathrm{del} 1}+\mathrm{mt}: \mathrm{Col}^{T 300 \mathrm{l}}$, and used expression of restriction enzyme to select against wild-type genome and used the temperature-dependent selection against the double mutant. When we expressed mito-Bglll in the germline while keeping the flies at $29^{\circ} \mathrm{C}$, about $14.6 \%$ of the $\mathrm{F} 1$ females were viable and fertile (Figure $4 \mathrm{~A}$ ). PCR analysis and restriction digestion showed that the progeny had lost the wild-type allele of $m t: N D 2$, which was targeted by the restriction enzyme, but they were heteroplasmic for a new $\mathrm{mt}: N D 2^{\text {del } 1}$ genome and the double-mutant parental $m t: N D 2^{\text {del1 }}+m t: C o{ }^{\text {T30ol }}$ genome (Figure 4A). Apparently, the wild-type mtDNA was cut and homologous repair or exchange using the mutant $m t: N D 2^{\text {del1 }}$ sequence led to loss of the site. Initially, the abundance of the newly generated mt:ND2del1 genome was very low in all the progeny (Figure 4B), probably because only a small fraction of the wild-type genomes underwent homologous exchange. However, because a low level of the recombinant genome is sufficient to rescue the temperature-sensitive phenotype of $m t: N D 2^{\text {del1 }}+m t: \mathrm{Col}^{T 300 l}$, this low level of recombinant genome

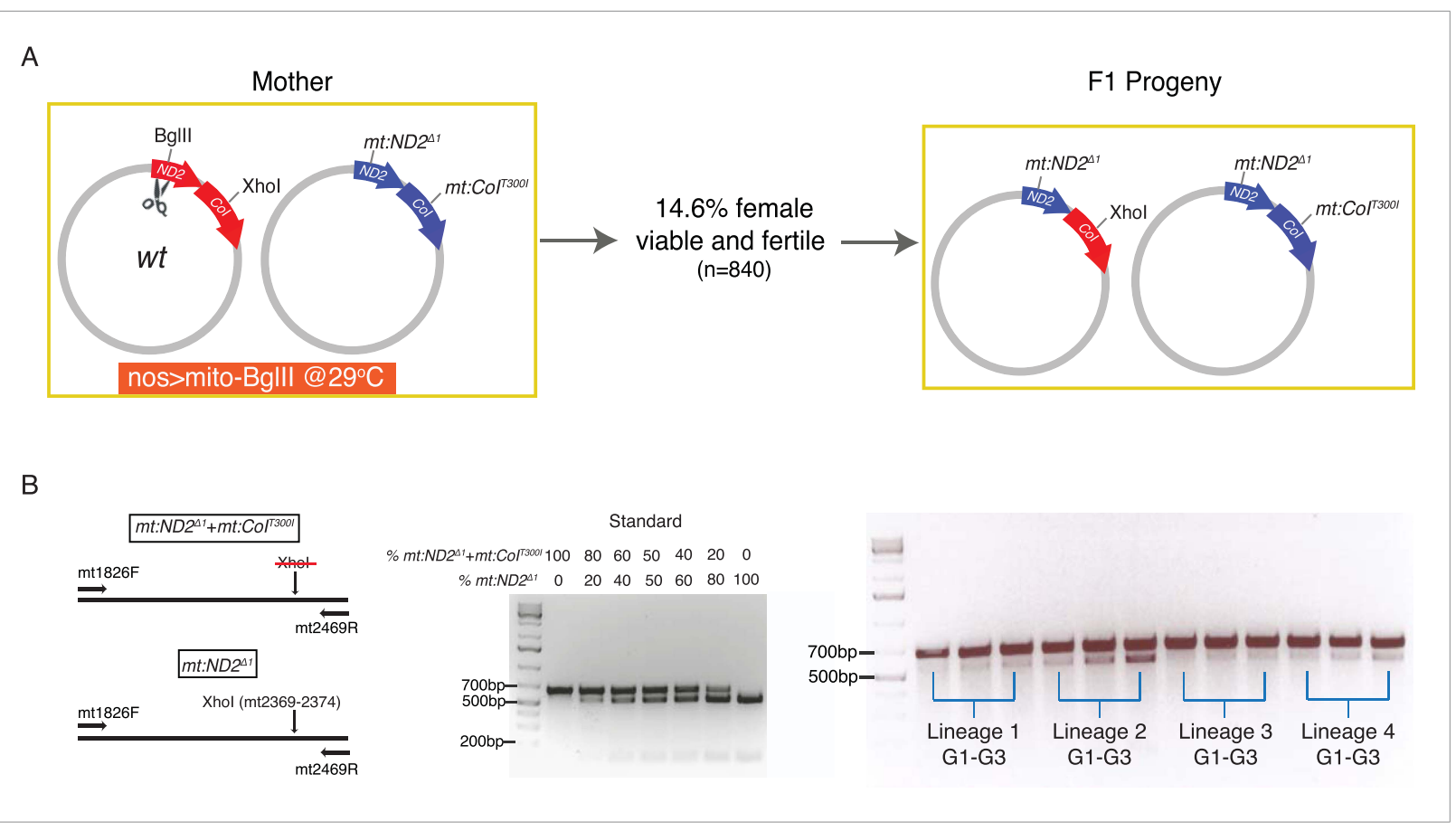

Figure 4. Homology-dependent conversion of the wild-type Bglll site into the sequence of the mt:ND2 ${ }^{\text {del1 }}$ allele following cutting of the Bglll site. (A) Expression of mito-Bglll in the germline of flies heteroplasmic for wild-type and $\mathrm{mt}: \mathrm{ND} 2^{\text {del1 }}+\mathrm{mt}$ : $\mathrm{Col}^{\text {T300I }}$ genomes at $29^{\circ} \mathrm{C}$ led to isolation of progeny with repaired wild-type genome at the Bglll site and converted it to mt:ND2 $2^{\text {del1 }}$. (B) The starting abundance for the newly generated mt:ND2 ${ }^{\text {del1 }}$ was low, but it increased over generations as the genome possessed a selective advantage at $29^{\circ} \mathrm{C}$. The abundance of mt:ND2 ${ }^{\text {del }}$ was measured by PCR amplifying a mtDNA region (mt1826-mt2496) using mtDNA from 40 adults as template followed by restriction digestion using Xhol in four heteroplasmic lineages from generation 1 to 3 .

DOI: 10.7554/eLife.07247.007 
supported production of fertile heteroplasmic flies. Since the more functional $m t: N D 2^{\text {del1 }}$ genome has a selective advantage at $29^{\circ} \mathrm{C}$, its abundance increased over subsequent generations (Figure $4 B$ ), and in some lineages, the $m t: N D 2^{d e l 1}+m t: C o l^{T 300 I}$ genome was completely eliminated after 18 generations (Ma et al., 2014).

Again, we isolated recombinant genomes at a relatively high frequency, which leads us to propose that a single DSB is sufficient to promote recombination (see 'Discussion'). However, we note that without additional markers, we could do little to characterize the nature of the exchange events.

\section{Exchange between the mitochondrial genomes of two different species}

In order to study whether DSB-induced exchanges involve restricted local repair events, or exchange of longer stretches of DNA, we needed more markers. To achieve this, we examined recombination between the diverged mitochondrial genomes of Drosophila melanogaster and Drosophila yakuba. We made a heteroplasmic D. melanogaster line in which the D. yakuba mtDNA is sensitive to Bglll and the D. melanogaster genome is sensitive to Xhol (see 'Materials and methods') (Figure 5A). Within the coding sequences, the D. yakuba genome shared about $93 \%$ sequence identity with mt:ND2 ${ }^{\text {del1 }}$, while the non-coding AT-rich sequence was highly diverged and reduced to about $1 \mathrm{~kb}$ in length. We introduced DSBs into both genomes by germline expression of mito-Bglll and mito-Xhol and found that about $10 \%$ of the females were fertile. Progeny homoplasmic for recombinant genomes were recovered. All propagating recombinants contained the AT-rich region of the D. melanogaster genome (assessed by Southern analysis, Figure 5-figure supplement 1), which we expected because $D$. melanogaster genomes outcompete $D$. yakuba genomes very quickly when co-resident ( $\mathrm{H}$ Ma and PH O'Farrell, unpublished). We characterized the mitochondrial genomes of three progeny lines by PCR and standard sequencing. All the recombinant genomes had one crossover point very close to but upstream of the Xhol site of the $m t: N D 2^{\text {dell }}$ genome (Figure $5 B$ ). A second crossover is considerably downstream: in two of the recombinants, the other crossover occurred between markers at mt3124 and 3184, and between markers at mt3379 and 3445. In the third case, the other crossover is further downstream and we obtained mixed sequencing signals for a region between mt5524 and mt6291 (Figure 5C). Flies were likely to be heteroplasmic for that particular region as if multiple recombinants are carried in this line. Based on these recombinants, we concluded that homologybased repair of DSBs promoted exchange of a substantial uninterrupted stretch of sequence. We also note that there were no discontinuities in the mapped region of exchange as might occur if repair randomly converted mismatches in heteroduplex in one direction or the other (see below for more discussion).

\section{Discussion}

This study, which provides direct evidence for homologous recombination in Drosophila mtDNA, opens up the possibility of recombinational mapping of functions on the mitochondrial genome in animals. While the details of recombinational mechanism remain to be worked out, our analysis outlines some of its features. If widespread in animals, the recombinational activity we have observed is likely to have a pervasive influence on the genetics and evolution of metazoan mitochondrial genomes.

\section{Features of mitochondrial recombination}

Multiple modes of homology-dependent exchange are used in different organisms and different situations. Though differing in their details, these different modes often involve DNA breaks of one or both strands, resection of ends, heteroduplex formation, local replication, and resolution. Some features of the processes contributing to mtDNA recombination can be inferred from the sequence of three D. melanogaster/D. yakuba recombinants that we isolated following introduction of DSBs.

In all cases, one crossover point was very close to the cleavage site of Xhol, indicating stimulation of exchange by DSBs. Some resection (8-44 bp) must have occurred as the sites of exchange are located two or three SNPs upstream of the cleavage site (Figure 5B). Surprisingly, the actual exchange events occur in sequences with frequent interruptions of homology such that one of the exchanges occurred within a stretch of only 11 bases of homology. In contrast, some of the more thoroughly characterized homology-dependent mechanisms such as those catalyzed by RecB-C and RecF require significantly greater homology (23-27 bp and 44-90 bp, respectively) (Shen and Huang, 1986). 
A

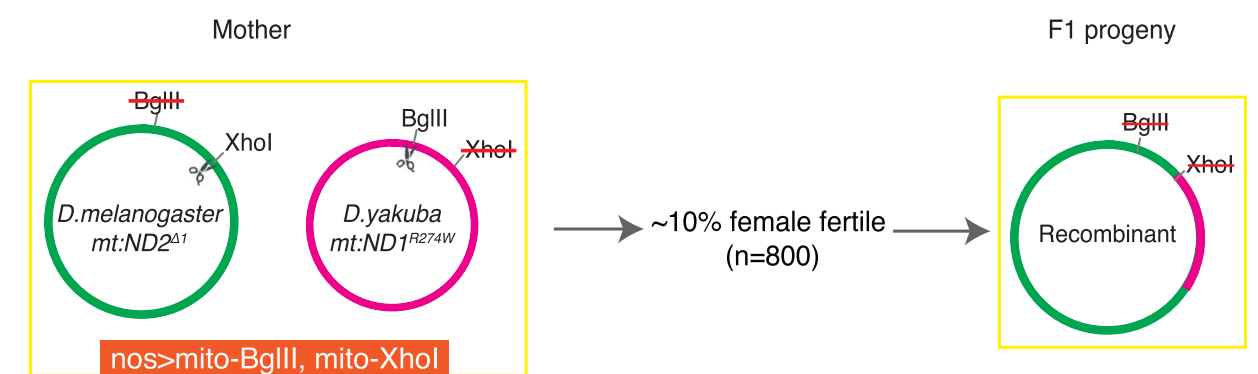

B

D. melanogaster genome: 2240>AATCAGGAAAAAAGGAAACTTTTGGTTCTCTAGGAATAATTTATGCTATA-TTAGCTATTGGATTATTAGGA>2310 D. yakuba genome: $\quad$ 2249>AATCTGGTAAAAAGGAAACTTTCGGTTCTTTAGGAATAATCTATGCTATACTT-GCTATTGGATTATTAGGA $>2319$ Recombinant genome 1: 2240>AATCAGGAAAAAAGGAAACTTTTGGTTCTCTAGGAATAATTTATGCTATA-TTAGCTATTGGATTATTAGGA>2310 Recombinant genome 2: $2240>$ AATCAGGAAAAAAGGAAACTTTTGGTTCTCTAGGAATAATTTATGCTATA-TTAGCTATTGGATTATTAGGA $>2310$ Recombinant genome 3: 2240>AATCAGGAAAAAAGGAAACTTTTGGTTCTCTAGGAATAATTTATGCTATA-TTAGCTATTGGATTATTAGGA>2310

$2311>$ TTTATTGTA TGAGCTCATCATATATTTAC C GTTGGAATAGA TGTAGATACTCGAGCTTATTTTACCTCAGCTACTATAATTATTGCAGTT>2400 2320>TTTATTGTT TGAGCTCATCATATATTTAC A GTTGGAATAGA CGTTGATACACGAGCTTATTTTACTTCTGCTACTATAATTATTGCGGTT>2409 $2311>$ TTTATTGTAIGGAGCTCATCATATATTTACAA GTTGGAATAGA CGTTGATACACGAGCTTATTTTACTTCTGCTACTATAATTATTGCGGTT>2400 2311> TTTATTGTA TGAGCTCATCATATATTTAC CIGTGGAATAGACGTTGATACACGAGCTTATTTTACTTCTGCTACTATAATTATTGCGGTT>2400 $2311>$ TTTATTGTA TGAGCTCATCATATATTTAC CIGTGGAATAGACGTTGATACACGAGCTTATTTTACTTCTGCTACTATAATTATTGCGGTT>2400

C

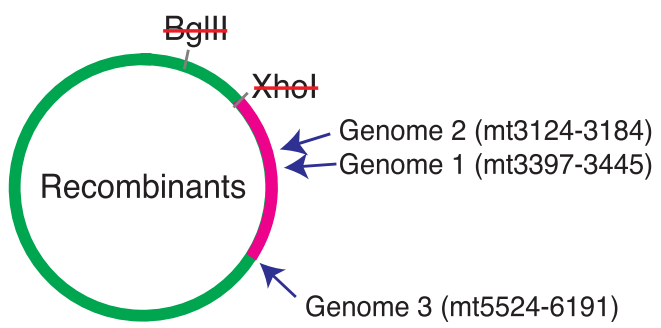

Figure 5. Exchange between two mitochondrial genomes of different species that share $93 \%$ sequence homology. (A) A heteroplasmic line containing both a Drosophila yakuba ( $m$ t:ND1 ${ }^{\text {R274m }}$ genome and a Drosophila melanogaster ( $m t: N D 2^{\text {del11 }}$ ) genome was made. $90 \%$ of heteroplasmic females induced to express both mito-Bglll and mito-Xhol enzymes in the germline were sterile, but the escaper progeny from the fertile females contained newly generated recombinant genotypes. (B) Sequences of the parental and three recombinant genomes reveal that one recombination junction is close to the Xhol site of the D. melanogaster genome. The Xhol site (yellow highlight) present in the D. melanogaster parent sequence (top line) is absent in all the other sequences. Pink rectangles indicate the interval in which recombination occurred. (C) A schematic illustration of where the second crossover was for the recombinant genomes. Arrows indicate the approximate position and the numbers, which are based on the $D$. melanogaster sequence, given the positions of bounding polymorphisms.

DOI: 10.7554/eLife.07247.008

The following figure supplement is available for figure 5 :

Figure supplement 1. Southern Blot analysis of the long EcoRI segment covering the AT-rich region of two parental genomes (D. yakuba mt:ND1R274W and D. melanogaster $m t: N D 2^{d e / 1}$ ) and three recombinant genomes.

This discordance favors other mechanisms such as break-induced recombination (BIR), a template switching mechanism of recombination, which can use very short regions of homology in an exchange process referred to as microhomology-mediated BIR (Hastings et alo, 2009; Anand et al., 2014), to be the underlying mechanism.

The second site of exchange is far from either cleavage site, but seems to occur in regions with high homology. It is not clear whether both exchanges occur at the same time: since the genomes are circular, some types of exchange will produce a dimer circle that might resolve by an intramolecular recombination event at a later time. Our finding that one of our recombinant lines carried more than 
one recombinant genome with a common point of exchange near the Xhol cleavage site but different downstream points of exchange is consistent with this possibility. If separated in time, propagation of the unresolved product of the first recombination could give a population of molecules and independent resolution events could give a mixed population. While these analyses give us only a limited perspective on the mechanism of recombination, it is notable that the abundant SNPs suggest that each of the characterized events involved exchange of a substantial segment without interruptions (at least $1 \mathrm{~kb}$ ), rather than restricted local repair.

When no DSBs were introduced by restriction enzymes in lines heteroplasmic for ATP6[1] and mt: $N D 2^{\text {del1 }}+\mathrm{mt}: \mathrm{Col}^{T 300}$, all of the recombinants again involved the exchange of a substantial and continuous stretch of sequence (at least $5 \mathrm{~kb}$ ), indicating either the exchange of extended region of duplex or priming of an extended stretch of replication template by a second genome. We also noted that two of the three recombinants had a crossover site very close to where the Drosophila mTERF (DmTTF) binds (mt6314-mt6341 and mt11698-mt11725, respectively [Roberti et al., 2003]). Although a relatively weak association, we call attention to it because mtDNA replication pauses at the DmTTF binding sites (Jõers et al., 2013), and such pauses might destabilize forks and promote exchange.

\section{The purpose of mitochondrial recombination}

Homologous recombination plays major roles in repairing DSBs and in genetic exchange. Below, we discuss the significance of these two roles for maintaining mitochondrial genomes in natural populations.

Our finding that restriction cutting gives frequent recombinant products demonstrates that this process is active in the repair of these DSBs in mtDNA. While genetically distinct genomes in heteroplasmic flies provide the means to detect homologous exchange, sibling molecules ought to have a greater opportunity to guide homology-dependent repair as they will occur, at least transiently, in the same mitochondrion or nucleoid. In homoplasmic flies, accurate repair using sibling molecules will leave no trace. Only misaligned intra-molecular recombination between repeats would give a clear signature. Occurrence of recombination junctions in regions of low homology in this study supports suggestions that non-allelic recombination can contribute to formation of deletions and duplications in the mitochondrial genomes-a frequent cause of mitochondrial defects during aging. Such a recombination could also explain several observations found in natural population (Rand and Harrison, 1989; La Roche et al., 1990; Lunt and Hyman, 1997; Ludwig et al., 2000). For instance, mtDNA of European rabbit (Oryctolagus councils) has repeated 153-bp motifs in the vicinity of the replication origin of $\mathrm{H}$ strand and very individual carries genomes with different numbers of these repeats (Casane et al., 1994). In Drosophila, mtDNA varies in size from 16 to $20 \mathrm{~kb}$ (Solignac et al., 1986; Townsend and Rand, 2004; Rand, 2011), mainly due to the variation in the length of the noncoding region, which contains two types of tandemly repeated elements (Lewis et al., 1994). Rand has shown that spontaneous changes in length in populations as if repeats can come and go at a relatively rapid pace (Rand, 2011). While accurate repair using sibling molecules is likely to be the main purpose of mitochondrial recombination, more rare non-allelic events can have a major impact on the production of variants, and exchanges between distinctly marked genomes, as reported here, provide a means to characterize the process.

Recombination is detected by and well known for its ability to promote genetic exchange. However, animal mtDNA is restricted in the types of exchange that can occur because uniparental inheritance prevents intermingling of genomes. The mitochondrial genome of each female is sheltered from encounters with foreign genomes and will be passed on in an isolated lineage or clone. Genetic exchange between distantly related genomes might occur as a result of occasional paternal transmission that introduces a foreign genome (e.g., Kraytsberg, 2004), but the significance of such violations of uniparental inheritance are matters of an ongoing debate. For the most part, the integrity of mitochondrial haplotypes suggests that the contribution of exchange among distantly related genomes is relatively small (Elson et al., 2001).

The limitations on genetic exchange imposed by uniparental inheritance, which are similar to those in asexually propagating organisms, do not mean that there is no meaningful genetic exchange (Hurst and Peck, 1996). It has become increasingly apparent that organisms can carry more than one mitochondrial genotype, that is, they are naturally heteroplasmic because of new mutations, 
stably transmitting heteroplamic combinations, or a breakdown of uniparental inheritance (Solignac et al., 1983; Ladoukakis et al., 2011; Tsang and Lemire, 2011; Payne et al., 2012; Ma et al., 2014; Ye et al., 2014). In this sense, the situation we created by introducing the temperature-sensitive genome into the ATP6[1] line, might be a model for things that can happen naturally. Emergence of a new genome with strong selfish drive, but with a defect in function, or introduction of such genome from another lineage will create a natural situation analogous to our experiment. The outcome of our experimental manipulation shows that this situation has generally detrimental consequences. Indeed, the temperature-sensitive mutation together with the drive advantage created a sort of 'population time bomb': the lineage remained healthy for multiple generations allowing the population to expand greatly, and then it collapsed upon elimination of the functional $\mathrm{mt}$ genome. Even rare recombination can uncouple a positively selected drive mutation from detrimental mutations, and as in our experiment, selection can then restore health. Thus, even occasional genetic exchange would prevent rogue genomes from wiping out lineages.

In conclusion, we show that recombination among mitochondrial genomes occurs in Drosophila and that this recombination can be used to manipulate genomes for functional mapping. We suggest that recombination will influence evolution of the mitochondrial genome in animals and impact the genetic behavior of mitochondrial disease mutations.

\section{Materials and methods}

\section{Experimental procedures}

Fly stocks

The homoplasmic stocks used in this study include the following mutant alleles: $m t: N D 2^{\text {del1 }}, \mathrm{mt}$ : $\mathrm{Col}^{R 3010}, \mathrm{mt}: \mathrm{Col}^{T 3001}$ and $\mathrm{mt}: \mathrm{ND}^{\text {del1 }}+\mathrm{mt}: \mathrm{Col}^{T 300 l} \cdot \mathrm{w}^{1118}$ was used to provide wild-type mtDNA. Flies homoplasmic for ATP6[1] mtDNA was kindly provided by Michael Palladino (University of Pittsburgh, U.S.). D. yakuba flies were obtained from Drosophila species stock center, San Diego. Other strains used included UAS-mito-BgIII, UAS-mito-Xhol, UAS-mito-Pstl, nos-Gal4, and ey-Gal4. The stocks were cultured at $18-25^{\circ} \mathrm{C}$ on standard fly medium.

\section{Generation of heteroplasmic flies}

Poleplasm transplantation was used to generate heteroplasmic flies, and the method was described in Ma et al. (2014). Basically, a portion of the poleplasm was sucked out from donor embryos and transferred into the posterior end of the recipient embryos. The injected recipient embryos were kept in a humidified chamber at $22^{\circ} \mathrm{C}$, and hatched larvae were transferred to vials with yeast paste in the next 2 days and incubated at $22^{\circ} \mathrm{C}$ until eclosion. Lines were established from the females obtained from the injected embryos, which were systematically mated to males with the recipient mtDNA genotype to ensure that the only source of newly introduced mitochondrial genomes was the injected material (i.e., that it did not arise from the purported possibility of exceptional paternal transmission). For each of these females, 10-30 F1 females were isolated to establish sublines. When not specified, each generation was derived from at least 50 individuals belonging to the previous generation.

The procedures to make the heteroplasmic line with both $D$. melanogaster (mt:ND2 ${ }^{\text {del1 }}$ ) and D. yakuba (mt:ND1 ${ }^{R 274 W}$ ) genomes involve the following four steps. Initially, in order to make a $D$. melanogaster line with the wild-type $D$. yakuba mitochondrial genome, cytoplasm from $D$. yakuba embryos was transplanted into the temperature lethal mutant $\left(m t: N D 2^{\text {del } 1}+m t: \mathrm{Col}^{\mathrm{T300}}\right)$ embryos, and eclosed adults were kept at $29^{\circ} \mathrm{C}$ to select for flies with the $D$. yakuba genome. By doing this, two independent lines were established and both stably transmitted $D$. yakuba mitochondrial genome (3-4\%) from generation to generation at $29^{\circ} \mathrm{C}$. Secondly, a mitochondrially targeted restriction enzyme, mito-Pstl, was expressed in the germline mitochondria of the two heteroplasmic lines to eliminate the $m t: N D 2^{\text {del1 }}+m t: \mathrm{Col}^{T 300 l}$ genome, as Pstl site was only present in the $\mathrm{D}$. melanogaster genome. Through this, several lines with only wild-type D. yakuba mtDNA were established. Surprisingly, D. melanogaster flies homoplasmic for $D$. yakuba mtDNA were as healthy as wild-type flies at both $22^{\circ} \mathrm{C}$ and $29^{\circ} \mathrm{C}$. Similar to the $D$. melanogaster genome, wild-type $D$. yakuba mtDNA has one Bglll site and one Xhol site. The Bglll site is located in the same place as in D. melanogaster's genome (mt:ND2), whereas the Xhol site, unlike the D. melanogaster genome (which is in the mt:Col coding region), is located in the mt:ND1 coding region further downstream. Thirdly, expression of mito-Xhol enzyme in the germline of flies with the wild-type $D$. yakuba genome was used to select 
a mutant derivative with an allele $m t: N D 1^{R 274 W}$ that removed the Xhol site. This led to a line that is sensitive to Bglll cut but not to Xhol. Finally, this line was then used as a recipient for making the $m t$ : $N D 2^{\text {del1 }} / \mathrm{mt}: N D 1^{R 274 W}$ heteroplasmic line.

\section{DNA isolation}

Genomic DNA was extracted from adults as described in Ma et al. (2014). In brief, flies were mechanically homogenized with a plastic pestle in homogenization buffer $(100 \mathrm{mM} \mathrm{Tris-HCl} \mathrm{[pH} \mathrm{8.8],}$ $0.5 \mathrm{mM}$ EDTA, $1.0 \% \mathrm{SDS}$ ). The homogenate was incubated at $65^{\circ} \mathrm{C}$ for $30 \mathrm{~min}$, followed by addition of potassium acetate (to $1 \mathrm{M}$ ) and incubation on ice $(30 \mathrm{~min})$ to precipitate protein and SDS. Subsequently, the homogenate was centrifuged at $20,000 \times \mathrm{g}$ for $10 \mathrm{~min}$ at $4^{\circ} \mathrm{C}$. DNA was recovered from the supernatant by adding $0.5 \mathrm{vol}$ of isopropanol and centrifuging at 20,000 $\times \mathrm{g}$ for $5 \mathrm{~min}$ at room temperature. The resultant pellet was washed with $70 \%$ ethanol and suspended in $100 \mu$ of $\mathrm{dd} \mathrm{H}_{2} \mathrm{O}$ per fly. mtDNA genotype frequencies were measured in individual founding females and their further generations via qPCR. When populations were analyzed, we extracted DNA from groups of 40 individuals.

\section{mtDNA isolation}

Unfertilized eggs were the best source for isolating pure mtDNA without much contamination from genomic DNA: each egg contains $10 \pm 2$ million copies of mtDNA, which provides about $0.2 \mathrm{ng}$ mtDNA but very little nuclear DNA. In order to isolate a large number of unfertilized eggs, virgins (XX) collected from flies with a certain mtDNA genotype were crossed to males with compound $X Y(C[1: Y])$. Because the progeny of such a cross were male sterile, bulk mating of the progeny gave an abundant source of unfertilized eggs. The unfertilized eggs were then collected and dechorionated with $50 \%$ bleach and washed before being homogenized in STE buffer $(100 \mathrm{mM} \mathrm{NaCl}, 50 \mathrm{mM}$ Tris- $\mathrm{HCl}$, $\mathrm{pH}=8.5,10 \mathrm{mM}$ EDTA) using Kimble-Chase Kontes 2-ml glass Dounce tissue grinder. The homogenate was supplemented with $1 \%$ SDS and proteinase $\mathrm{K}$ and incubated at $55^{\circ} \mathrm{C}$ for $1 \mathrm{hr}$. The sample was further treated with RNase $A$ at $37^{\circ} \mathrm{C}$ for $30 \mathrm{~min}$ before phenol-chloroform extraction and ethanol precipitation.

\section{Southern blotting}

Southern blotting was used to detect the recombinant mitochondrial genome. It was performed as described in Ma et al. (2014). Basically, digested DNA was separated on a $0.8 \%$ agarose gel by electrophoresis and transferred to Hybond $\mathrm{N}+$ membrane by the capillary method. DNA transferred to the membrane was fixed by UV cross-linking (Stratalinker $120 \mathrm{~mJ}$ ). The blot was hybridized with PCR-generated probes (mt1577-mt2365) that were labeled with DIG-11-dUTP using $0.35 \mathrm{mM}$ DIG-11-dUTP, $1.65 \mathrm{mM}$ dTTP, and Bioline Velocity Taq DNA polymerase, following the manufacturer's instructions (Roche, Germany). Prehybridization and hybridization were carried out at $41^{\circ} \mathrm{C}$ overnight in DIG Easy Hyb buffer solution (Roche). The membrane was washed two times with $2 \times \mathrm{SSC}+0.1 \% \mathrm{SDS}$ at room temperature for $5 \mathrm{~min}$ and twice for $15 \mathrm{~min}$ with $0.1 \times$ SSC $+0.1 \%$ SDS at $65^{\circ} \mathrm{C}$. Hybridized membrane was visualized with NBT/BCIP following the manufacturer's instructions (Roche). mtDNA primers used to generate the DIG-labeled probe are listed in Supplementary file 2.

\section{Sequencing mtDNA via PacBio SMRT technique}

Due to a long repetitive sequence in the AT-rich non-coding region of Drosophila mtDNA, PacBio SMRT was used to sequence the whole mitochondrial genome for three genotypes. SMRT sequencing can generate extraordinary long reads (>30,000 bp) with extremely high consensus accuracy (>99.999\%). Thus, the whole AT rich region could be covered in a single read without the trouble of re-assembly. Purified mtDNA (isolated as described above) was linearized by restriction cutting at the Pstl site and a $\sim 20-\mathrm{kb}$ band was isolated and gel purified after electrophoresis. About $500 \mathrm{ng}$ of gel purified linear mtDNA sample was used for library preparation using a modified $10 \mathrm{~kb}$ Template Preparations Protocol (PacBio, University of Washington, Seattle). Basically, the blunt hairpin SMRTBell adaptors were ligated to the repaired ends of the double-strand DNA fragments. Failed ligation products were removed by adding Exolll and ExoVII exonucleases. The attached templates were further purified with $0.5 \times$ and $0.45 \times$ bead-washes for sequencing. We conducted the sequencing reactions on a PacBio RSIl system using one SMRT cell for each genome. All libraries were sequenced using P4/C2 chemistry. mtDNA samples from $m t: N D 2^{\text {del1 }}+m t: \mathrm{Col}^{T 300 l}$, and the recombinants were 
run with $120 \mathrm{~min}$ movie time and ATP6[1] sample was run with $180 \mathrm{~min}$. Raw reads were analyzed following either HGAP (for de novo assembly) and BLASR protocol (for resequencing) in SMRT Portal 2.2. The coverage for each samples ranged from $1000 \times$ to $35,000 \times$.

When only the coding region of some genomes were sequenced, two long-range PCR reactions using Expand Long Template PCR system (Roche) was performed: mt186-mt7502, mt6905-mt14797 with the following program: 1 cycle of $93^{\circ} \mathrm{C}$ for $3 \mathrm{~min}, 30$ cycles of $93^{\circ} \mathrm{C} 15 \mathrm{~s}, 50^{\circ} \mathrm{C} 30 \mathrm{~s}, 60^{\circ} \mathrm{C} 8 \mathrm{~min}$, and 1 cycle of $60^{\circ} \mathrm{C}$ for $10 \mathrm{~min}$. Primers were designed all around the coding region (Supplementary file 2) for sequencing by QuintaraBio (Albany, CA).

\section{Acknowledgements}

This research was supported by NIH (GM086854) funding to PHO'F. HM was supported by the Longterm postdoc fellowship from Human Frontiers Science Program. We thank Hong Xu for generously sharing unpublished information and reagents, and Michael J Palladino at University of Pittsburgh for kindly providing us flies with ATP6[1] genome. We also are grateful for support from the PacBio team, particularly Nicole Rapicavoli (Melon Park, CA), Maika Malig (University of Washington, Settle), and Roberto Lleras.

\section{Additional information}

Funding

\begin{tabular}{lll} 
Funder & Grant reference & Author \\
\hline NIH Office of the Director & GM086854 & Patrick H O'Farrell \\
\hline Human Frontier Science Program & LT000138/2010-L & Hansong Ma \\
\hline
\end{tabular}

The funders had no role in study design, data collection and interpretation, or the decision to submit the work for publication.

Author contributions

HM, Conception and design, Acquisition of data, Analysis and interpretation of data, Drafting or revising the article; $\mathrm{PHO}$ 'F, Conception and design, Analysis and interpretation of data, Drafting or revising the article

Author ORCIDs

Patrick H O'Farrell, (D) http://orcid.org/0000-0003-0011-2734

\section{Additional files}

Supplementary files

- Supplementary file 1. HR-based repair was also able to rescue in somatic tissues like eye. High-level eye-antennal disc expression of both mito-Bglll and mito-Xhol using ey-Gal4 at $29^{\circ} \mathrm{C}$ resulted in pupal lethality (headless pupa) in wild-type flies or in flies carrying $\mathrm{mt}$ genomes resistant to only one of the two enzymes. A few (about 1\%) escapers eclosed and the majority of eclosed flies had no eye or small eye phenotype (rows 1 and 2). Having both genomes increased the eclosion rate to 10\% (row 3) and most of the eclosed flies had normal-sized eyes (not shown).

DOI: 10.7554/eLife.07247.010

- Supplementary file 2. A list of primers used in this study.

DOI: $10.7554 /$ LLife.07247.011

\section{References}

Anand RP, Tsaponina O, Greenwell PW, Lee CS, Du W, Petes TD, Haber JE. 2014. Chromosome rearrangements via template switching between diverged repeated sequences. Genes \& Development 28:2394-2406. doi: 10. 1101/gad.250258.114.

André C, Levy A, Walbot V. 1992. Small repeated sequences and the structure of plant mitochondrial genomes.

Trends in Genetics 8:128-132. 
Bacman SR, Williams SL, Moraes CT. 2009. Intra- and inter-molecular recombination of mitochondrial DNA after in vivo induction of multiple double-strand breaks. Nucleic Acids Research 37:4218-4226. doi: 10.1093/nar/ gkp348.

Bender A, Krishnan KJ, Morris CM, Taylor GA, Reeve AK, Perry RH, Jaros E, Hersheson JS, Betts J, Klopstock T, Taylor RW, Turnbull DM. 2006. High levels of mitochondrial DNA deletions in substantia nigra neurons in aging and Parkinson disease. Nature Genetics 38:515-517. doi: 10.1038/ng1769.

Berlin S, Smith NG, Ellegren H. 2004. Do avian mitochondria recombine? Journal of Molecular Evolution 58 : 163-167. doi: 10.1007/s00239-003-2537-z.

Birky CW. 1995. Uniparental inheritance of mitochondrial and chloroplast genes: mechanisms and evolution. Proceedings of the National Academy of Sciences of USA 92:11331-11338. doi: 10.1073/pnas.92.25.11331.

Cao Z, Wanagat J, McKiernan SH, Aiken JM. 2001. Mitochondrial DNA deletion mutations are concomitant with ragged red regions of individual, aged muscle fibers: analysis by laser-capture microdissection. Nucleic Acids Research 29:4502-4508. doi: 10.1093/nar/29.21.4502.

Casane D, Dennebouy N, de Rochambeau H, Mounolou JC, Monnerot M. 1994. Genetic analysis of systematic mitochondrial heteroplasmy in rabbits. Genetics 138:471-480.

Celotto AM, Chiu WK, Van Voorhies W, Palladino MJ. 2011. Modes of metabolic compensation during mitochondrial disease using the Drosophila model of ATP6 dysfunction. PLOS ONE 6:e25823. doi: 10.1371/ journal.pone.0025823.

Celotto AM, Frank AC, McGrath SW, Fergestad T, Van Voorhies WA, Buttle KF, Mannella CA, Palladino MJ. 2006. Mitochondrial encephalomyopathy in Drosophila. The Journal of Neuroscience 18:810-820. doi: 10.1523/ JNEUROSCI.4162-05.2006.

Ciborowski KL, Consuegra S, García de Leániz C, Beaumont MA, Wang J, Jordan WC. 2007. Rare and fleeting: an example of interspecific recombination in animal mitochondrial DNA. Biology Letters 3:554-557. doi: 10.1098/ rsbl.2007.0290.

Cortopassi GA, Shibata D, Soong NW, Arnheim N. 1992. A pattern of accumulation of a somatic deletion of mitochondrial DNA in aging human tissues. Proceedings of the National Academy of Sciences of USA 89: 7370-7374. doi: 10.1073/pnas.89.16.7370.

DeLuca SZ, O'Farrell PH. 2012. Barriers to male transmission of mitochondrial DNA in sperm development. Developmental Cell 22:660-668. doi: 10.1016/j.devcel.2011.12.021.

Dujon B, Slonimski PP, Weill L. 1974. Mitochondrial genetics IX: a model for recombination and segregation of mitochondrial genomes in Saccharomyces cerevisiae. Genetics 78:415-437.

Elson JL, Andrews RM, Chinnery PF, Lightowlers RN, Turnbull DM, Howell N. 2001. Analysis of European mtDNAs for recombination. American Journal of Human Genetics 68:145-153. doi: 10.1086/316938.

Fukui H, Moraes CT. 2009. Mechanisms of formation and accumulation of mitochondrial DNA deletions in aging neurons. Human Molecular Genetics 18:1028-1036. doi: 10.1093/hmg/ddn437.

Guo XH, Liu SJ, Liu Y. 2006. Evidence for recombination of mitochondrial DNA in triploid crucian carp. Genetics 172:1745-1749. doi: 10.1534/genetics.105.049841.

Hagström E, Freyer C, Battersby BJ, Stewart JB, Larsson N-G. 2013. No recombination of mtDNA after heteroplasmy for 50 generations in the mouse maternal germline. Nucleic Acids Research 42:1111-1116. doi: 10. 1093/nar/gkt969.

Hastings PJ, Ira G, Lupski JR. 2009. A microhomology-mediated break-induced replication model for the origin of human copy number variation. PLOS Genetics 5:e1000327. doi: 10.1371/journal.pgen.1000327.

Hill JH, Chen Z, Xu H. 2014. Selective propagation of functional mitochondrial DNA during oogenesis restricts the transmission of a deleterious mitochondrial variant. Nature Genetics 46:389-392. doi: 10.1038/ng.2920.

Hurst LD, Peck JR. 1996. Recent advances in understanding of the evolution and maintenance of sex. Trends in Ecology \& Evolution 11:A46-A52. doi: 10.1016/0169-5347(96)81041-X.

Jõers P, Lewis SC, Fukuoh A, Parhiala M, Ellilä S, Holt IJ, Jacobs HT. 2013. Mitochondrial transcription terminator family members mTTF and mTerf5 have opposing roles in coordination of mtDNA synthesis. PLOS Genetics 9 : e100380. doi: 10.1371/journal.pgen.1003800.

Kraytsberg Y. 2004. Recombination of human mitochondrial DNA. Science 304:981. doi: 10.1126/science. 1096342.

La Roche J, Snyder M, Cook DI, Fuller K, Zouros E. 1990. Molecular characterization of a repeat element causing large-scale size variation in the mitochondrial DNA of the sea scallop Placopecten magellanicus. Molecular Biology and Evolution 7:45-64.

Ladoukakis ED, Theologidis I, Rodakis GC, Zouros E. 2011. Homologous recombination between highly diverged mitochondrial sequences: examples from maternally and paternally transmitted genomes. Molecular Biology and Evolution 28:1847-1859. doi: 10.1093/molbev/msr007.

Ladoukakis ED, Zouros E. 2001. Direct evidence for homologous recombination in mussel (Mytilus galloprovincialis) mitochondrial DNA. Molecular Biology and Evolution 18:1168-1175. doi: 10.1093/ oxfordjournals.molbev.a003904.

Lewis DL, Farr CL, Farquhar AL, Kaguni LS. 1994. Sequence, organization, and evolution of the A+T region of Drosophila melanogaster mitochondrial DNA. Molecular Biology and Evolution 11:523-538.

Liu VW, Zhang C, Nagley P. 1998. Mutations in mitochondrial DNA accumulate differentially in three different human tissues during ageing. Nucleic Acids Research 26:1268-1275. doi: 10.1093/nar/26.5.1268.

Ludwig A, May B, Debus L, Jenneckens I. 2000. Heteroplasmy in the mtDNA control region of sturgeon (Acipenser, Huso and Scaphirhynchus). Genetics 156:1933-1947.

Lunt DH, Hyman BC. 1997. Animal mitochondrial DNA recombination. Nature 387:247. doi: 10.1038/387247a0. 
Ma H, Xu H, O'Farrell PH. 2014. Transmission of mitochondrial mutations and action of purifying selection in Drosophila melanogaster. Nature Genetics 46:393-397. doi: 10.1038/ng.2919.

Matsuura ET, Chigusa SI, Niki Y. 1989. Induction of mitochondrial DNA heteroplasmy by intra- and interspecific transplantation of germ plasm in Drosophila. Genetics 122:663-667.

Mita S, Rizzuto R, Moraes CT, Shanske S, Arnaudo E, Fabrizi GM, Koga Y, DiMauro S, Schon EA. 1990.

Recombination via flanking direct repeats is a major cause of large-scale deletions of human mitochondrial DNA. Nucleic Acids Research 18:561-567. doi: 10.1093/nar/18.3.561.

Muller HJ. 1932. JSTOR: The American Naturalist, Volume 66. p. 118-138.

Payne BA, Wilson IJ, Yu-Wai-Man P, Coxhead J, Deehan D, Horvath R, Taylor RW, Samuels DC, Santibanez-Koref M, Chinnery PF. 2012. Universal heteroplasmy of human mitochondrial DNA. Human Molecular Genetics 22: 384-390. doi: 10.1093/hmg/dds435.

Rand DM. 2011. Population genetics of the cytoplasm and the units of selection on mitochondrial DNA in Drosophila melanogaster. Genetica 139:685-697. doi: 10.1007/s10709-011-9576-y.

Rand DM, Harrison RG. 1989. Molecular population genetics of mtDNA size variation in crickets. Genetics 121: 551-569.

Rank GH, Bech-Hansen NT. 1972. Somatic segregation, recombination, asymmetrical distribution and complementation tests of cytoplasmically-inherited antibiotic-resistance mitochondrial markers in S. cerevisiae. Genetics 72:1-15.

Roberti M, Polosa PL, Bruni F, Musicco C, Gadaleta MN, Cantatore P. 2003. DmTTF, a novel mitochondrial transcription termination factor that recognises two sequences of Drosophila melanogaster mitochondrial DNA. Nucleic Acids Research 31:1597-1604. doi: 10.1093/nar/gkg272.

Sato A, Nakada K, Akimoto M, Ishikawa K, Ono T, Shitara H, Yonekawa H, Hayashi J. 2005. Rare creation of recombinant mtDNA haplotypes in mammalian tissues. Proceedings of the National Academy of Sciences of USA 102:6057-6062. doi: 10.1073/pnas.0408666102.

Sato M, Sato K. 2013. Maternal inheritance of mitochondrial DNA by diverse mechanisms to eliminate paternal mitochondrial DNA. Biochimica et Biophysica Acta 1833:1979-1984. doi: 10.1016/j.bbamcr.2013.03.010.

Shedge V, Arrieta-Montiel M, Christensen AC, Mackenzie SA. 2007. Plant mitochondrial recombination surveillance requires unusual RecA and MutS homologs. The Plant Cell 19:1251-1264. doi: 10.1105/tpc.106. 048355.

Shen P, Huang HV. 1986. Homologous recombination in Escherichia coli: dependence on substrate length and homology. Genetics 112:441-457.

Solignac M. 2004. Mitochondrial DNA in the Drosophila melanogaster complex. Genetica 120:41-50. doi: 10. 1023/B:GENE.0000017628.52056.97.

Solignac M, Monnerot M, Mounolou JC. 1983. Mitochondrial DNA heteroplasmy in Drosophila mauritiana. Proceedings of the National Academy of Sciences of USA 80:6942-6946. doi: 10.1073/pnas.80.22.6942.

Solignac M, Monnerot M, Mounolou JC. 1986. Concerted evolution of sequence repeats in Drosophila mitochondrial-DNA. Journal of Molecular Evolution 24:53-60. doi: 10.1007/BF02099951.

Townsend JP, Rand DM. 2004. Mitochondrial genome size variation in New World and Old World populations of Drosophila melanogaster. Heredity 93:98-103. doi: 10.1038/sj.hdy.6800484.

Tsang WY, Lemire BD. 2011. Stable heteroplasmy but differential inheritance of a large mitochondrial DNA deletion in nematodes. Biochemistry and Cell Biology 80:645-654. doi: 10.1139/o02-135.

Ujvari B, Dowton M, Madsen T. 2007. Mitochondrial DNA recombination in a free-ranging Australian lizard. Biology Letters 3:189-192. doi: 10.1098/rsbl.2006.0587.

Xu H, DeLuca SZ, O'Farrell PH. 2008. Manipulating the metazoan mitochondrial genome with targeted restriction enzymes. Science 321:575-577. doi: 10.1126/science.1160226.

Ye K, Lu J, Ma F, Keinan A, Gu Z. 2014. Extensive pathogenicity of mitochondrial heteroplasmy in healthy human individuals. Proceedings of the National Academy of Sciences of USA 111:10654-10659. doi: 10.1073/pnas. 1403521111.

Zassenhaus HP, Denniger G. 1994. Analysis of the role of the NUC1 endo/exonuclease in yeast mitochondrial DNA recombination. Current Genetics 25:142-149. doi: 10.1007/BF00309540. 